\title{
Divertor power load feedback with nitrogen seeding in ASDEX Upgrade
}

\author{
A. Kallenbach, R. Dux, J.C. Fuchs, R. Fischer, B. Geiger, L. \\ Giannone, A. Herrmann, T. Lunt, V. Mertens, R. McDermott, \\ R. Neu, T. Pütterich, S. Rathgeber, V. Rohde, K. Schmid, J. \\ Schweinzer, W. Treutterer, ASDEX Upgrade Team \\ Max-Planck-Institut für Plasmaphysik, EURATOM Association, Garching, Germany
}




\begin{abstract}
Feedback control of the divertor power load by means of nitrogen seeding has been developed into a routine operational tool in the all-tungsten clad ASDEX Upgrade tokamak. For heating powers above about $12 \mathrm{MW}$, its use has become inevitable to protect the divertor tungsten coating under boronised conditions. The use of nitrogen seeeding is accompanied by improved energy confinement due to higher core plasma temperatures, which more than compensates the negative effect of plasma dilution by nitrogen on the neutron rate. This paper describes the technical details of the feedback controller. A simple model for its underlying physics allows the prediction of its behaviour and to optimize the feedback gain coefficients used. Storage and release of nitrogen in tungsten surfaces was found to have substantial impact on the behaviour of the seeded plasma, resulting in increased nitrogen consumption with unloaded walls and a latency of nitrogen release over several discharges after its injection. Nitrogen is released from tungsten plasma facing components with moderate surface temperature in a sputtering-like process, therefore no uncontrolled excursions of the nitrogen wall release are observed. Overall, very stable operation of the high- $Z$ tokamak is possible with nitrogen seeding, where core radiative losses are avoided due to its low atomic charge $\mathrm{Z}$ and a high ELM frequency is maintained.
\end{abstract}

\title{
1. Introduction
}

For a burning plasma device like ITER, radiative power removal by seed impurities will be inevitable to avoid divertor damage by excessive heat flux [1] [2]. To avoid long time fuel storage by co-deposition, only recycling impurities like nitrogen or noble gases can be used as extrinsic radiators. Impurity seeding has been applied to fusion devices since the 1980s [3]. Many early studies were devoted to effects of confinement improvement, connected to a peaking of the density profile. Improved confinement following impurity seeding in radiating mantle discharges was reported from TEXTOR (RI-mode [4]), ASDEX Upgrade (CDH-mode [5]) and DIII-D [6]). During Ar injection into JT-60U [7], some of the effects observed in smaller machines were reproduced, namely confinement improvement and a moderate peaking of the density profile. Pronounced density peaking occured in seeding of advanced tokamak plasmas in JT-60U [8]. However, it turned out that the mechanisms leading to improved confinement appeared not to be active under all conditions. Nitrogen injection studies in the EDA H-mode in Alcator C-Mod [9] showed moderately reduced confinement $\left(\mathrm{H}_{\text {ITER89p }}\right.$ decreasing from 1.9 to about 1.6), while almost vanishingly small target power loads could be achieved. Radiative divertor experiments with Ar in DIII-D showed little effect on the energy confinement [10]. Despite extensive experimental efforts, energy confinement in JET has so far been observed to degrade with impurity injection [11] or to remain constant [12] [13] [14]. Some improvement was found for 'afterpuff' conditions, [15] [16], demonstrating the complex interplay of deuterium and impurity puffing. Radiative fractions above 0.8 could be achieved in JT-60U long pulse ELMy H-mode discharges with Ar injection, albeit with $\mathrm{H}$-factors $\mathrm{H}_{98(y, 2)}$ in the range 0.87-0.75 [17].

In early studies, the impurities were injected either in a pre-programmed trajectory [7] or via feedback-control using measurements of the total radiated power [18], an 
impurity spectral line, or a selected edge bolometer chord [9]. Such schemes become less appropriate in high-power devices with low intrinsic radiation, where impurity seeding becomes a requirement to avoid divertor power overload. This situation occured in ASDEX Upgrade after the removal of all carbon plasma facing components (PFCs) [19] and wall conditioning by boronization [20]. For JET, the same is expected after installation of the ITER like wall (ILW) in 2011, for which a lot of preparatory work with impurity seeding had been performed recently [21]. To effectively protect the divertor from excessive heat loads, a feedback signal is required which is closely related to the target power load. In principle, a real-time calculation of the difference in heating power and radiated power or a real-time thermography system would be suitable candidates. The sensor complexity and the required complicated calculations to derive the heat load are critical to a system relevant for machine safety. In ASDEX Upgrade, a feedback signal has been selected, that supplies an indirect measurement of the divertor load and which is very simple and robust in real-time data aquisition and evaluation: the (thermo) electric current into a divertor tile, measured as voltage at a shunt resistor embedded in the tile mounting. This feedback system has been used routinely on ASDEX Upgrade since the first boronization with all-tungsten PFCs [20], using nitrogen as the seeding species. Figure 1 shows two video images of comparable discharges without and with nitrogen cooling. The reduction of divertor tile edge glowing due to the nitrogen seeding is clearly visible. Besides the desired effect of target heat load reduction, smaller ELMs with higher frequency and improved confinement have been observed [22]. This beneficial effect makes the scheme more attractive, but is not a major subject of this study.

This paper is focussed on the technical implementation of the feedback system implemented in ASDEX Upgrade and the understanding and optimizations of the used Proportional-Integral (PI) controller. The shunt measurement of the (thermo-)electric current into the outer divertor target [23] probes mainly the temperature difference [24] between the outer and inner divertor (or the upper divertor in case of near doublenull). Since the inner divertor has very low electron temperatures in between ELMs, the thermo-electric current corresponds in good appproximation to the outer divertor temperature. In fact, good correlation between the measured electric current and the electron temperature measured by Langmuir probes in the scrape-off layer close to the strike point was found for H-mode conditions. Since the interpretation of the measured signal in terms of a divertor temperature appeared more useful for operation and interpretation, the current is multiplied by a fixed factor obtained by comparison to Langmuir probe data and termed $T_{d i v}$ throughout this paper, with units of eV. For H-mode conditions, $T_{d i v}$ was found to also represent a reasonable approximation of the outer divertor peak power load (see next section). To understand and further optimise the feedback system and its control gains, an off-line model has been developed, which includes a simple physics model for the plasma response on the impurity injection.

This paper is organized as follows. Section 2 decribes the pre-processing of the sensor signal, which is required to remove the contribution of ELMs. The PI controller 

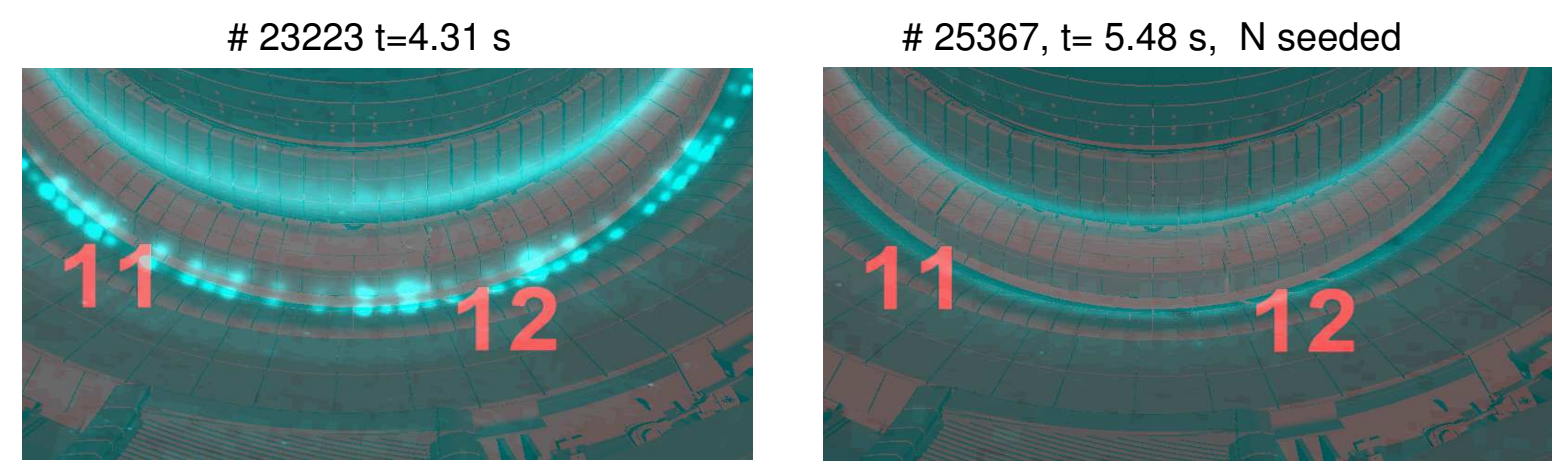

Figure 1. Video observation of the divertor for boronised high-power H-mode discharges with $14 \mathrm{MW}$ total heating power without and with nitrogen seeding. The emission pattern (light blue) overlayed on the machine CAD view (black and red) represents thermal surface radiation in the near-IR and some contribution of visible light emission. $H_{\alpha}$ radiation is mostly blocked by a filter. The structured emission at the outer target without nitrogen seeding is largely attributed to local tile overheating. Numbers indicate the toroidal sector.

is introduced in section 3. A simple 0-D plasma response model is developed in section 4 that allows the prediction of the divertor behaviour in response to the impurity gas puff. For the case of nitrogen and a tungsten wall, this model has to take into account the storage and release of seeded atoms from the tungsten wall surface. The realistic simulation of the plasma response allows the optimization of the feedback gains and to test different initialization conditions, shown in section 5 . The paper is concluded by a brief summary of the results.

\section{Pre-processing for ELM removal}

Divertor temperature and target power load during H-modes are strongly modulated by ELMs. Radiative cooling mostly controls the inter-ELM power load, but additional control tools are required to account for the ELM size and frequency. To obtain direct and undisturbed control of the inter-ELM heat load, ELMs have to be removed from the sensor signal, which is sampled at a rate of $100 \mathrm{kHz}$. This is realised by application of a modified median filter in the LabVIEW RT real-time data aquisition [25], as shown in figure 2. The filter is adapted to the typical ELM frequency $(50-300 \mathrm{~Hz})$ and duration range ( $0.5-3 \mathrm{~ms})$ in ASDEX Upgrade. The filter takes the $100^{\text {th }}$ largest of the 700 data points recorded in the previous $7 \mathrm{~ms}$, thus eliminating large ELM contributions and also negative spikes in the electric current observed during the ELM start time. The filter supplies the ELM-cleaned signal $T_{d i v}$ to the discharge control system with a cycle time of $1 \mathrm{~ms}$, while the control system itself operates with a cycle time of $1.5-2 \mathrm{~ms}$. The total time delay of the acknowledged $T_{\text {div }}$ value is not longer than $2 \mathrm{~ms}$, compared to an off-line calculation.

The sensor data $T_{d i v}$ is in fact a good represention of the inter-ELM peak power flux 
density, $P_{\max }$, in the outer divertor, which is the actual quantity to be controlled for machine protection. Figure 2 shows a monotonic and approximately linear relationship between $T_{d i v}$ and $P_{\max }$. The data with cold divertor conditions and good confinement are obtained with nitrogen seeding.
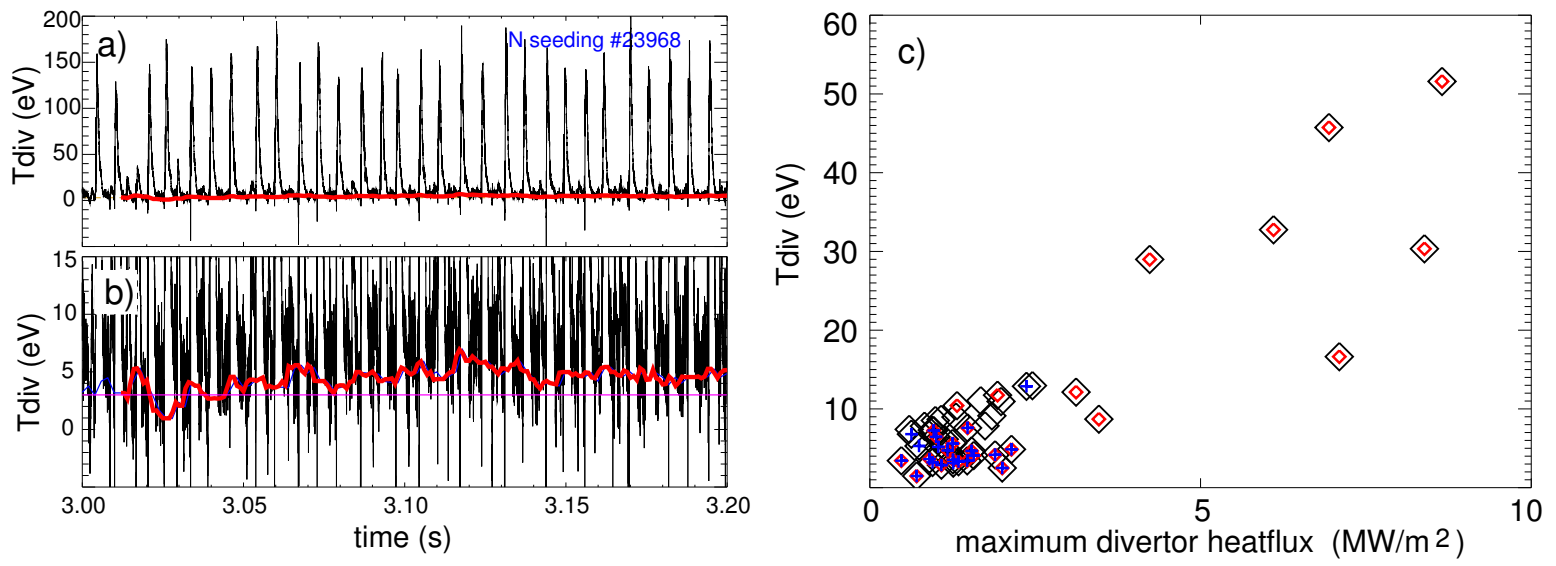

Figure 2. a) Thermoelectric current signal at a poloidal tile row at the outer target (black), multiplied by $0.02 \mathrm{eV} / \mathrm{A}$ to obtain approximate divertor temperature as measured by Langmuir probes, and $T_{\text {div }}$ obtained by unsymmetric median filtering $\left(100^{\text {th }}\right.$ largest of 700 values). The median filter is very effective in removing the ELM contribution (thick red line). b) same with expanded scale. c) $T_{\text {div }}$ vs. the inter-ELM peak power load in the outer divertor, obtained by median filtering of IR thermography data, for various $H$-mode discharges $\left(I_{p}=1 M A, B_{t}=-2.5 \mathrm{~T}\right)$ with different heating powers and with and without nitrogen seeding. Data points marked with red diamonds have confinement factor $H_{98 y, 2}>0.9$. A central blue cross marks data points with nitrogen seeding.

\section{PI controller for nitrogen puff rate}

The nitrogen puff rate is determined by means of a PI controller in the real-time control system [26]. The core of the feedback algorithm is described by equation 1 for the nitrogen valve flux $\Phi_{\text {valve }}$ using the difference between set and actual divertor temperature, $T_{d i f f}=T_{\text {set }}-T_{\text {div }}$, where $T_{\text {div }}$ is proportional to the median filtered target current.

$$
\Phi_{\text {valve }}=\Phi_{f f}+K_{p} \cdot T_{\text {diff }}+\Phi_{\text {integral }}
$$

The contribution of the integral part, $\Phi_{\text {integral }}$, is calculated for timepoint t as

$$
\Phi_{\text {integral }}=\Phi_{i}^{0}+K_{i} \cdot \int_{\text {tstart }}^{t} T_{\text {diff }} d t
$$



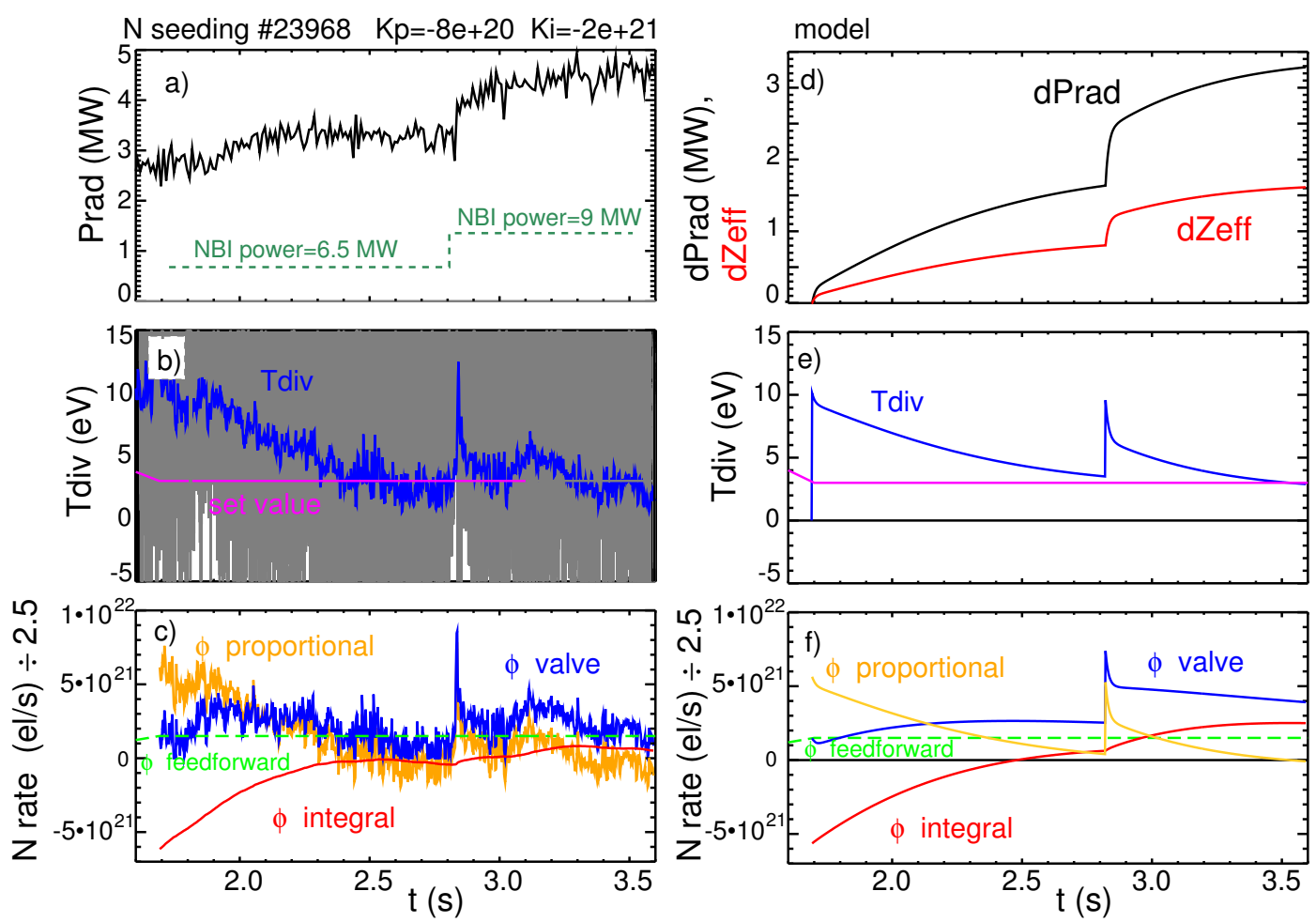

Figure 3. Experimental time traces (a-c) and simulation (d-f) for a discharge with feedback controlled nitrogen seeding (see section 4). a) plasma radiation, b) $T_{\text {div }}$, c) Nitrogen puff rate and the individual contributions to the controller output. The actual gas puff is about a factor 2.5 larger than assumed by the feedback controller since the divertor valves were not calibrated for nitrogen in this early pulse. $d, e, f$ ) show the corresponding parameters obtained from the plasma model, in addition the predicted $Z_{\text {eff }}$ rise due to nitrogen is given in d).

$\mathrm{K}_{p}$ and $\mathrm{K}_{i}$ are the feedback gain coefficients for the proportional and integral parts, $\Phi_{i}^{0}$ is the initial value of $\Phi_{\text {integral }}$ when the feedback starts. The coefficients have negative numerical values since increased impurity puffing reduces the target power load. It is useful to divide the output flux of the feedback system into the fluxes resulting from the proportional and integral parts, as shown in figure 3. Here, an additional small feedforward flux, $\Phi_{f f}$, which had been introduced to optimise the feedback performance, is also seen. Such a feedforward flux is no longer regarded as necessary after final optimisation of the gains.

For the initialisation of the controller, the start value of the integral part, $\Phi_{i}^{0}$, is important. There are two possible alternatives. First, using $\Phi_{i}^{0}=-K_{p} \cdot T_{\text {diff }}($ tstart $)$, the gas flux remains at feedback switch-on, either zero or on its feedforward value. This ensures a smooth transition into the controlled discharge phase. The second choice is to set the integral part to zero at switch-on-time, thus starting with the proportional part of the gas valve set flow. This results in a quicker approach to the $T_{\text {set }}$ value, but may lead to larger initial plasma perturbation. 
The values of the gain coefficients largely determine the behaviour of the system. The absolute values have to be chosen according to the desired output gas flux value. The proportional part, $\mathrm{K}_{p}$, is responsible for quick reaction to transients, the integral part, $\mathrm{K}_{i}$, determines how fast and how accurately the set value is reached. The ratio $K_{p} / K_{i}$ is the time constant for the equilibrium approach of the controller and should compensate the slowest of the dominant physics reaction time constants, which is in this case the pumping time. A good first guess for the coefficients is obtained as follows. We assume a hot divertor transient with $T_{\text {diff }}=10 \mathrm{eV}$ and a reasonable maximum nitrogen flux (discounting a H-L transition) is $\Phi_{\text {valve }}=1 \cdot 10^{22} \mathrm{el} / \mathrm{s}$. From this, a first estimate leads to $K_{p}=-110^{21} \mathrm{el} / \mathrm{s} / \mathrm{eV}$. Note that we use electrons per $\mathrm{s}$ in the units for the gain coefficients.

The time constants for radiation build-up and pumping are about 50 and $200 \mathrm{~ms}$, respectively. Aiming for a time constant $K_{p} / K_{i}$ comparable to the longer pumping time, the corresponding $K_{i}$ is $-510^{21} \mathrm{el} / \mathrm{s} /(\mathrm{s} \cdot \mathrm{eV})$. If gases other than nitrogen are used for radiative cooling, both $\mathrm{K}_{p}$ and $\mathrm{K}_{i}$ have to be scaled linearly down with the relative radiative capability of the seed gas compared to nitrogen. The radiative cooling rate $\Delta \mathrm{P}_{\text {rad }} / \mathrm{n}_{Z}$ scales roughly as $\mathrm{Z}^{3}$ for core radiation [27]. Reasonable values for $\mathrm{K}_{p, i}$ are obtained by scaling $\propto 1 / Z^{3}$, resulting in about a factor 3 lower gains for neon and a factor 17 lower gains for argon in comparison to nitrogen. The output valve flux is clipped to a maximum allowed value. In the case of clipping the integral part is frozen to avoid the accumulation of a too large integral value.

The gains should be optimised to achieve a fast and stable approach to the set value, to avoid large excursions of the flux, and to produce an almost constant valve flux during quasi-stationary discharge phases. Higher gains result in quicker reactions, but have the danger of over-shooting or even oscillatory behaviour. This is studied in section 5 using the off-line plasma model to optimise the sytem.

\section{Simple plasma response model for PI gain optimisation}

To allow off-line predictions of the feedback system behaviour, a model for the response of the divertor temperature (in terms of the electric current) to the seed gas flow is needed. The model is divided into three steps. First, the nitrogen content of the plasma and divertor is calculated using the valve flow rate and the pumping speed. Second, the radiative power loss is determined using the nitrogen density and a simple radiation model. Finally, the divertor temperature and thermoelectric current (the feedback sensor) are derived from an empirical scaling using the net power flux entering the divertor. The response obtained by the simple plasma model is plotted on the right hand side of figure 3, where the different contributions of the proportional and integral parts of the valve flux are also shown. Since wall storage of nitrogen turned out to have a major impact on particle balance, this had to be included into the plasma response model and is decribed separately in section 4.2. Finally, the choice of model parameters and the performance of the model are discussed with respect to a pair of ASDEX Upgrade 
discharges with and without nitrogen seeding. While the plasma response model is formulated for arbitrary seed impurities specified by their atomic charge Z, significant wall storage is only expected for nitrogen, therefore the storage model only applies to nitrogen.

\subsection{Particle balance, radiation and divertor temperature}

The nitrogen content of the main chamber and divertor is calculated using a simple particle balance model. A fixed distribution of the seed atoms between the main chamber and divertor is assumed, which is described by a simple chamber model [28]. Parameters are the divertor nitrogen enrichment factor $\eta_{N}$, the main plasma and divertor volumes $\mathrm{V}_{\text {main }}$ and $\mathrm{V}_{\text {div }}$, the pumping speed $\mathrm{S}_{N}$, the experimental parameters average plasma density $n_{D, \text { main }}^{i}$ and neutral divertor deuterium density $\mathrm{n}_{D, d i v}^{0}$. The superscripts ${ }^{i}$ and ${ }^{0}$ refer to ions and neutrals. We assume ions to constitute the main chamber particle content and neutrals the main divertor content. The compression factor $\mathrm{c}$ for a species is the ratio of its divertor density and its main chamber density. The nitrogen enrichment is the ratio of the nitrogen and deuterium compression factors $\mathrm{c}_{N, D}, \eta_{N}=c_{N} / c_{D}=\left(n_{N, \text { div }}^{0} / n_{N, \text { main }}^{i}\right) /\left(n_{D, \text { div }}^{0} / n_{D, \text { main }}^{i}\right)$. Since the divertor neutral nitrogen content is not directly measured (and hence the nitrogen pumping rate), estimates for $\eta_{N}$ are used in combination with the measured main chamber ion densities and the deuterium neutral divertor density to determine $n_{N, d i v}^{0} . \eta$ can be determined experimentally from the decay rate of the impurity content after the injection phase [28] or from 2-d divertor modelling [29]. The first method is clearly disturbed by strong nitrogen wall pumping and release, while 2-d modelling results for nitrogen are not yet available. We use $\eta_{N}=2$, an estimate obtained by adapting the $\eta_{A r}$ derived from $\mathrm{Ar}$ decay time measurements under very similar experimental conditions. $\eta_{N}$ is one of the least known parameters in this study, we estimate its uncertainty to $\pm 50 \%$.

The other relevant ASDEX Upgrade parameters are $\mathrm{V}_{\text {main }}=14 \mathrm{~m}^{3}, \mathrm{~V}_{d i v}=1.5 \mathrm{~m}^{3}, \mathrm{~S}_{N}=$ $20 \mathrm{~m}^{3} / \mathrm{s}$. The discharges used for this paper have a line-averaged electron density $\bar{n}_{e}=$ $810^{19} \mathrm{~m}^{-3}$ and $n_{D, d i v}^{0}=3.510^{20}$ atoms $/ \mathrm{m}^{3}$.

Assuming stiff coupling of main chamber and divertor densities, an effective volume,

$\mathrm{V}_{\text {eff }}$, can be calculated to obtain the nitrogen density in the main chamber $n_{N, \text { main }}^{i}$ from the total number of atoms, $N=N_{N, \text { main }}+N_{N, \text { div }}, n_{N, \text { main }}^{i}=N / V_{\text {eff }}$

$$
V_{e f f}=V_{\text {main }}\left(1+N_{N, \text { div }} / N_{N, \text { main }}\right)=V_{\text {main }}+c_{N} V_{\text {div }}
$$

Similarly, the number of pumped nitrogen atoms can be expressed by simple algebraic transformations in terms of $\mathrm{N}$ and $\mathrm{V}_{\text {eff }}$ :

$$
\Phi_{\text {pump }}=S_{N} \cdot n_{N, d i v}^{0}=S_{N} \cdot N_{N, d i v} / V_{d i v}=S_{N} \cdot N \cdot c_{N} / V_{e f f}
$$

The time dependent nitrogen content of the main chamber plus divertor is then obtained by integrating the equation for the total number of nitrogen atoms

$$
d N / d t=\Phi_{\text {valve }}-\Phi_{\text {pump }}-\Phi_{\text {wall }}
$$


where $\Phi_{\text {valve }}$ is the nitrogen valve flux in atoms/s. $\Phi_{\text {wall }}$ is the net nitrogen flux taken up by the wall and will be further described in the next section. While the pumping speed $S_{N}$ applies to molecules, which are the dominant nitrogen species in the pump chamber, no factor of 2 appears in equation 5 since the pumped nitrogen flux is also in atoms/s.

The simple model used to describe the particle distribution between main chamber and divertor is valid as long as the pumping time is long compared to transfer times from SOL to divertor and divertor to SOL [30]. Under these conditions, the recycling pattern, and also the radiation distribution, is determined by the internal time constants and does not depend on the puff location. If the divertor enrichment gets large, or the pumping speed is very high, the simple 2-chamber approximation breaks down and divertor puffing can indeed lead to higher divertor radiation in comparison to core puffing. Such conditions are observed in the DIII-D puff and pump scenario [10], where the strike point is situated very close to the pump throat. Our model neglects core particle transport (treated as infinitely fast) and radial profile shape effects.

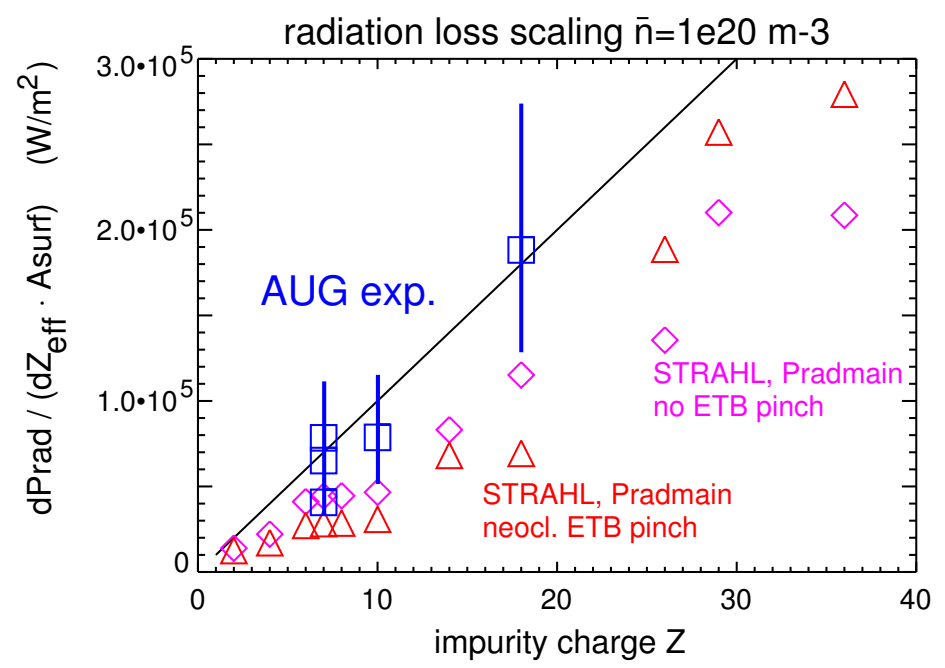

Figure 4. Normalized radiative loss for different impurity species, characterised by their atomic charge $Z$. Data represented by squares have been derived from radiatively cooled $A S D E X$ Upgrade $H$-mode discharges. Triangles and diamonds represent calculations with the 1-d impurity transport code STRAHL using atomic data from ADAS. Two different transport models have been used, the effect of ELMS on the radiation has not been taken into account in the STRAHL calculations. The solid line corresponds to the prediction of equation 6 with $f_{\text {rad }}=110^{-36} \mathrm{Wm}^{4}$.

Having specified the impurity density in the main chamber and divertor, a simple scaling is used to calculate the additional radiative power loss caused by the seed impurity. In the following, approximate formulas for arbitrary seed species are given, defined by the atomic charge number $\mathrm{Z}$. N stands for the total number of impurity atoms in the system. Similar to the ansatz in [31] we use

$$
d P_{\text {rad }}=f_{\text {rad }} Z \bar{n}_{e}^{2} A_{\text {surf }} d Z_{\text {eff }}
$$


with the $Z_{\text {eff }}$ increase due to the seed species with charge $\mathrm{Z}$

$$
d Z_{e f f}=Z(Z-1) N /\left(V_{e f f} \bar{n}_{e}\right)
$$

$A_{\text {surf }}$ is the plasma surface area. The linear $\mathrm{Z}$ dependence of the incremental radiation in equation 6 differs from the multi-machine scaling result [31], where a weak $\mathrm{Z}$ dependence was found for the global plasma radiation behaviour. Impurity transport and radiation modelling has been carried out for the incremental radiation of various species with the STRAHL code [32]. The results are shown in figure 4. Calculations with and without the assumption of a neoclassical inward pinch in the edge transport barrier region predict an approximately linear $\mathrm{Z}$ dependence of the additional radiation per $\mathrm{Z}_{\text {eff }}$ increase. For the lower $\mathrm{Z}$ species, the STRAHL calculations underestimate the radiative losses by about a factor 2 since divertor radiation is not calculated in this code. The normalisation factor $f_{\text {rad }}$ introduced in equation 6 has been calibrated against typical AUG H-mode discharges with medium gas puff and heating power, we obtain $f_{\text {rad }}=110^{-36} \mathrm{Wm}^{4}$. This value is compatible to the STRAHL results since the latter do not include divertor radiation. Since $\mathrm{dZ}_{\text {eff }}$ is proportional to $\mathrm{Z}(\mathrm{Z}-1) \approx \mathrm{Z}^{2}$, the total incremental radiated power scales approximately with $Z^{3}$ for constant impurity density and plasma parameters.

Finally, the divertor temperature and thermo-electric current have to be calculated using the power flux into the divertor. We use a modified version of an ELM averaged empirical scaling for AUG [23],

$$
\begin{aligned}
& T_{\text {div }, \text { scale }}=f_{\text {Tdiv }} \cdot\left(\bar{n}_{e} / n_{\text {Greenwald }}\right)^{-2} \cdot\left(P_{\text {div }}-P_{\text {detach }}\right) / R, P_{\text {div }}>P_{\text {detach }} \\
& 0, P_{\text {div }}<P_{\text {detach }}
\end{aligned}
$$

where $P_{d i v}=\mathrm{P}_{\text {heat }}-\mathrm{P}_{\text {rad,intrinsic }}-\mathrm{P}_{\text {rad,seed }}$ is the power flux to the divertor target, expressed as the difference of the heating power $P_{\text {heat }}$ and the intrinsic and seeded radiated power contributions. $P_{\text {detach }}$ is the power where $T_{d i v}$ becomes 0 , which is associated with power detachment of the outer divertor. $\mathrm{R}$ is the major radius of the machine and $n_{\text {Greenwald }}$ the Greenwald density, which has a value of about $1.210^{20} \mathrm{~m}^{-3}$ for the present conditions. For typical H-mode conditions in AUG, the inner divertor is detached in between ELMs and plasma heat deposition occurs almost exclusively at the outer target. $f_{\text {Tdiv }}=310^{-6}[\mathrm{eV} \mathrm{m} / \mathrm{W}]$ is a normalisation constant derived from AUG measurements. For the modelling of the seeded discharge, $\mathrm{P}_{\text {heat }}-\mathrm{P}_{\text {rad,intrinsic }}$ is either taken from a reference pulse without seeding or approximated by a simple expression for the radiated power, such as $\mathrm{P}_{\text {rad,intrinsic }}=0.4 \cdot \mathrm{P}_{\text {heat }}$, as it is typical for the discharges in this paper. The introduction of $\mathrm{P} / \mathrm{R}$ in the scaling (equation 8 ) was motivated by its planned application to other devices, e.g. ITER, and the assumption that $P / R$ is a divertor similarity parameter [33]. 


\subsection{Wall storage of nitrogen}

During nitrogen seeding experiments with tungsten plasma facing components, significant wall storage of nitrogen has been observed. This is made exceedingly clear by subsequent discharges after nitrogen seeding experiments, in which substantial nitrogen content of the plasma is measured (see figure 5). To investigate the influence of nitrogen wall storage on the feedback and to predict possible drawbacks on operation, the simple plasma response model is extended to include a model for wall storage and release of nitrogen.

The nitrogen wall storage model is based on recent laboratory measurements, in which a tungsten surface was bombarded by a $\mathrm{N}_{2}^{+}$ion beam [34]. Target biasing allowed the nitrogen impact energy to be varied up to $50 \mathrm{eV}$ per atom, which is the expected range for the conditions at tokamak surfaces. A saturation of the tungsten surface was observed in the lab experiments at a level of $810^{19} \mathrm{~N}$ atoms $/ \mathrm{m}^{2}$, corresponding roughly to a 1:1 atomic number ratio of tungsten to nitrogen over a depth of $1.8 \mathrm{~nm}$. The nitrogen is believed to be bound in a WN nitride phase, resulting in $50 \%$ nitrogen atomic concentration. The depth corresponds to the penetration depth of nitrogen according to TRIDYN simulations. The saturation can therefore be explained by the low diffusivity of nitrogen in tungsten for low temperature conditions. The WN nitride phase is expected to be stable for temperatures below $600 \mathrm{~K}$, in accordance with experimental observations of a stable tungsten content in tokamak surfaces over days without plasma operation.

The laboratory experiments were done with very smooth and clean surfaces (tungsten deposited on silicon wafers). We use the qualitative behaviour reported in [34] as a starting point to fit the experimental data obtained in ASDEX Upgrade tokamak discharges. The assumption of saturation of wall storage results in a sticking coefficient

$S_{c}$ dependent on the nitrogen surface areal density, $D_{\text {nitrogen }}=N_{\text {nitrogen }, \text { wall }} / A_{\text {wall }}$. N is the number of nitrogen atoms and $A_{\text {wall }}$ the surface area of the corresponding wall element (limiter or divertor). The sputtering yield of nitrogen, $\mathrm{Y}_{N}$, is assumed to rise linearly with low nitrogen surface areal density and to adopt a constant value beyond the saturation density. For simplicity, both quantities are described by an exponential ansatz which satisfies the boundary conditions and limits as discussed above. For the sticking coefficient, we use

$$
S_{c}=S_{0} \exp \left(-D_{N} / D_{N, s a t}\right)
$$

with $S_{0}=0.7$ to take into account reflection of the nitrogen atoms from a clean tungsten surface according to TRIM calculations [34]. The sticking approaches the value of zero for high nitrogen surface densities. This means that nitrogen entering the tungsten surface is either quickly lost from the surface or another nitrogen atom gets displaced. The nitrogen saturation areal density used to model the temporal behavior of the nitrogen flux for unboronised ASDEX Upgrade conditions is $\mathrm{D}_{N, \text { sat }}=110^{21}$ atoms $/ \mathrm{m}^{2}$. This value is an order of magnitude larger than the value found for an almost ideal tungsten surface in the lab experiment [34]. A possible explanation for this difference is the much higher surface roughness of the tokamak tungsten coating, or the storage 
of nitrogen in impurity layers (boron, carbon) residing in the valleys of the surface structure. An effective rise of the surface area due to roughness by a factor of 10 would reduce the derived saturation areal density by the same factor, bringing it close to the lab result obtained for a smooth surface.

The sputtering of nitrogen out of the near-surface layers is described by the ansatz

$$
Y_{N}=Y_{N, s a t}\left(1-\exp \left(-D_{N} / D_{N, s a t}\right)\right)
$$

This gives a sputtering rate that is linear with the nitrogen surface density at low nitrogen concentrations and a saturated value for high concentrations. AUG experiments suggest a value $Y_{\text {nitrogen,sat }}=0.05$ with reference to the impinging deuterium ion flux. This value includes the effect of sputtering of nitrogen by impurities, which have a higher yield, but a low impinging flux. For a realistic simulation of nitrogen storage in AUG, both main chamber walls and divertor surfaces have to be taken into account. The total nitrogen fluxes to both regions are comparable. However, due to the larger main chamber area this region determines the long-term storage, while the divertor exhibits a stronger influence on short time scales.

To calculate the nitrogen fluxes to and from the wall, first the deuterium recycling fluxes need to be specified. The divertor deuterium ion flux is taken from the experiment, since no simple scaling expression is available. We use $\Phi_{D \text {,div }}=410^{22} \mathrm{ion} / \mathrm{s}$ as measured by Langmuir probes, which is valid for all discharges in this paper where the same deuterium gas puff of $810^{21}$ atoms/s was used. The wetted divertor surface area is $A_{d i v}=$ $0.5 \mathrm{~m}^{2}$. The main chamber fluxes generally have a complicated pattern [35] and different recycling areas need to be taken into account. These include the 12 outboard limiters (about $1 \mathrm{~m}^{2}$ total wetted area), the inner heat shield $\left(9 \mathrm{~m}^{2}\right)$ and a region between the lower inner heat shield and the inner divertor, where strong neutral recycling is usually observed. Since the recycling pattern is not routinely measured, estimates or a scaling relation have to be used to calculate the total main chamber wall deuterium ion flux. A scaling relation based on global plasma parameters derived from AUG and JET [36] yields, for the experimental conditions of this paper, a midplane recycling flux density of $1.810^{21} \mathrm{D}$ atoms/s. This value multiplied by an effective surface area of $10 \mathrm{~m}^{2}$ gives a main chamber wall flux of $1.810^{22} \mathrm{D}$ atoms/s, corresponding to slightly more than two times the injected valve flux. To take into account the recycling at the lower inner heat shield, this value is multipied by 1.5 , arriving at a total wall flux of $\Phi_{D, \text { main }}=2.7$ $10^{22} \mathrm{D}$ atoms/s. The uncertainty of this value is estimated to be a factor of 2 . Since the assumed flux is distributed over different wall elements, an effective wetted surface area needs to be defined. We use $A_{\text {main }}=3 \mathrm{~m}^{2}$ as the effective wetted main chamber surface.

The nitrogen flux entrained in the deuterium flux is assumed to be proportional to the nitrogen concentration in the main plasma, but enhanced by the enrichment factor $\eta_{N}$ both for the divertor and main chamber wall fluxes. This enrichment is a consequence of the ionisation length of nitrogen being shorter than that of deuterium [29]. Finally, the main chamber and divertor walls are introduced into the nitrogen particle balance 

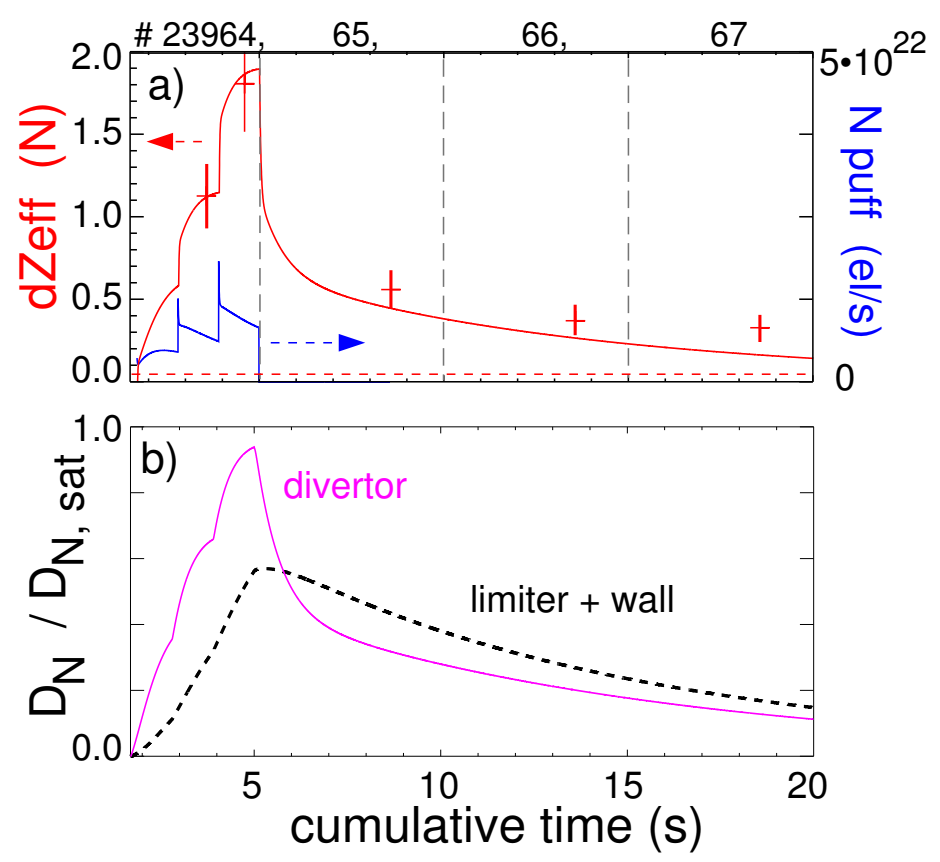

Figure 5. a) Lines show the model calculation of the plasma nitrogen content due to wall sticking. Pulse 23964 had feedback controlled nitrogen seeding starting at $1.7 \mathrm{~s}$. From 4 to $5 \mathrm{~s}$ the nitrogen puff was extraordinarily high due to a radiation induced L-mode phase. The subsequent $3 \mathrm{H}$-mode pulses were run with the same parameters but without nitrogen injection. These pulses were treated in the modelling as consecutive $5 \mathrm{~s}$ flattop phases, so effects during plasma current ramp-up and rampdown are omitted. The crosses show the plasma $Z_{\text {eff }}$ contribution of nitrogen measured by CXR spectroscopy at $\rho_{p}=0.7$. The dashed line indicates the typical intrinsic nitrogen $Z_{\text {eff }}$ contribution of $0.042\left(\epsilon_{N}=0.1 \%\right)$. b) shows the degree of nitrogen surface saturation, $D_{N} / D_{N, \text { sat }}$ for divertor and main chamber limiters.

equation 5 using the plasma nitrogen concentration $\epsilon_{N}=N_{\text {nitrogen,main }} /\left(\bar{n}_{e} V_{\text {main }}\right)$ and the SOL/divertor enrichment $\eta_{N}$ :

$$
\begin{aligned}
& \Phi_{\text {wall }}=\epsilon_{N} \eta_{N} \Phi_{D, \text { main }} S_{c, \text { main }} \\
& \quad+\epsilon_{N} \eta_{N} \Phi_{D, \text { div }} S_{c, \text { div }} \\
& \quad-\Phi_{D, \text { main }} Y_{N, \text { main }}-\Phi_{D, \text { div }} Y_{N, \text { div }}
\end{aligned}
$$

$S_{c, \text { main }}$ and $S_{c, \text { div }}$ are the nitrogen sticking coefficients for main chamber and divertor according to equation 9 .

The nitrogen decay is not reproduced perfectly in figure 5, however, its qualitative evolution is clear from the wall storage model: surfaces with low area and high fluence, like the divertor target, have quick re-erosion and deliver an immediate, high nitrogen release, which lasts only for a short period. Larger surface areas, like limiters or the inner heat shield, have a much slower release rate, but determine the long-term behaviour.

After about 10 discharges (50 plasma seconds) the nitrogen content has returned to its intrinsic level of about $0.1 \%$. The storage behaviour as shown in figure 5 is 
permanent at room temperature without plasma: A similar nitrogen level has been observed in the first discharge after a day without plasma experiments and with full torus pumping. There is obviously no significant thermal release of implanted nitrogen from tungsten under room temperature conditions. The fact that the release term for nitrogen out of tungsten has the magnitude of a sputtering coefficient ( $\mathrm{Y}_{N}$ in the order of a few $10^{-2}$ of the deuterium flux) ensures that no catastrophic, large flux release of nitrogen occurs. Such a rise of the nitrogen plasma content without corresponding valve flux has so far not been observed on AUG. The sputtering yield $\mathrm{Y}_{N}$ used to simulate the AUG experiments is much larger than typical sputtering yields observed for tungsten, suggesting that the nitrogen release is not only caused by the erosion of surface material as inferred from lab experiments with a pure nitrogen beam [34]. The higher erosion under tokamak conditions may be explained by the additional deuterium flux or by transient heating of surface layers during ELMs.

\subsection{Calibration and testing of the plasma response model}

The simple 0-D plasma response model described before contains a few 'free' parameters, for which experimental measurements were required to determine their values. This was done using a number of AUG pulses with and without nitrogen seeding. The coefficients describing the wall storage behaviour are largely determined by the long-term storage and release behaviour, as shown in the previous section.
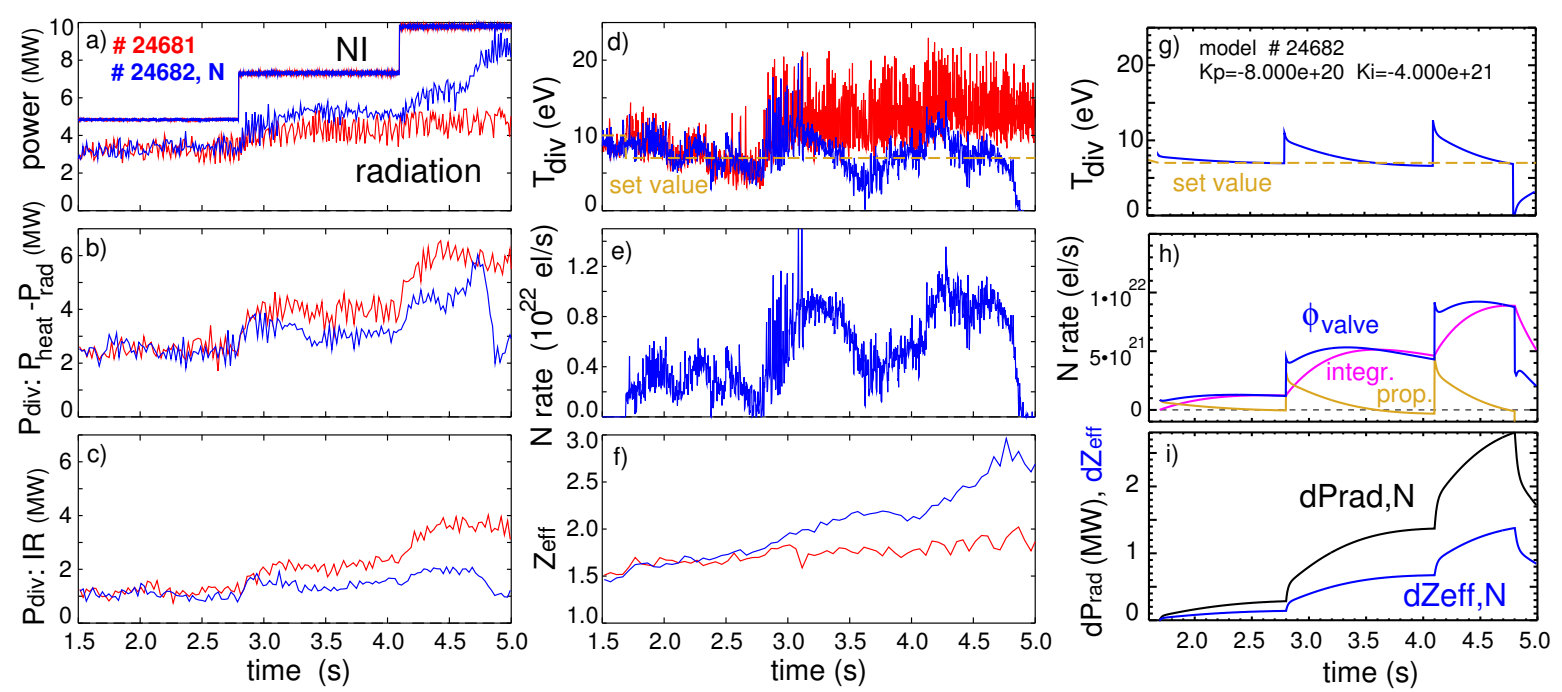

Figure 6. a)-f) Experimental data for a pair of discharges with (blue) and without (red) nitrogen seeding feedback control for unboronised conditions. a) NBI heating power and total radiation, b) difference of total heating power and radiated power, $c$ ) power measured by IR thermography at the divertor strike regions, $d$ ) sensor signals in terms of $T_{\text {div }}$, nitrogen puff rate for the seeded discharge, $f$ ) line-averaged $Z_{\text {eff }}$ from bremsstrahlung measurements. Boxes g)-i) show the corresponding predictions of the simple plasma model. 
Figure 6 compares measurements and model predictions for a pair of discharges with and without seeding. The numerical solution for the response model is obtained by simple forward-integration of equation 5 , with the flux contributions given by equations 1, 4, 11 and parameters calculated bq equations 6-9. The time step-width of the AUG control system of $2 \mathrm{msec}$ was found to be sufficient to obtain numerically stable results. This would allow an easy future integration of the response model into the feedback control system. It should be pointed out here that the plasma response model clearly does not have the quality of an integrated tokamak model. However, it is supposed to give a realistic input for feedback simulation or tokamak flight simulator applications.
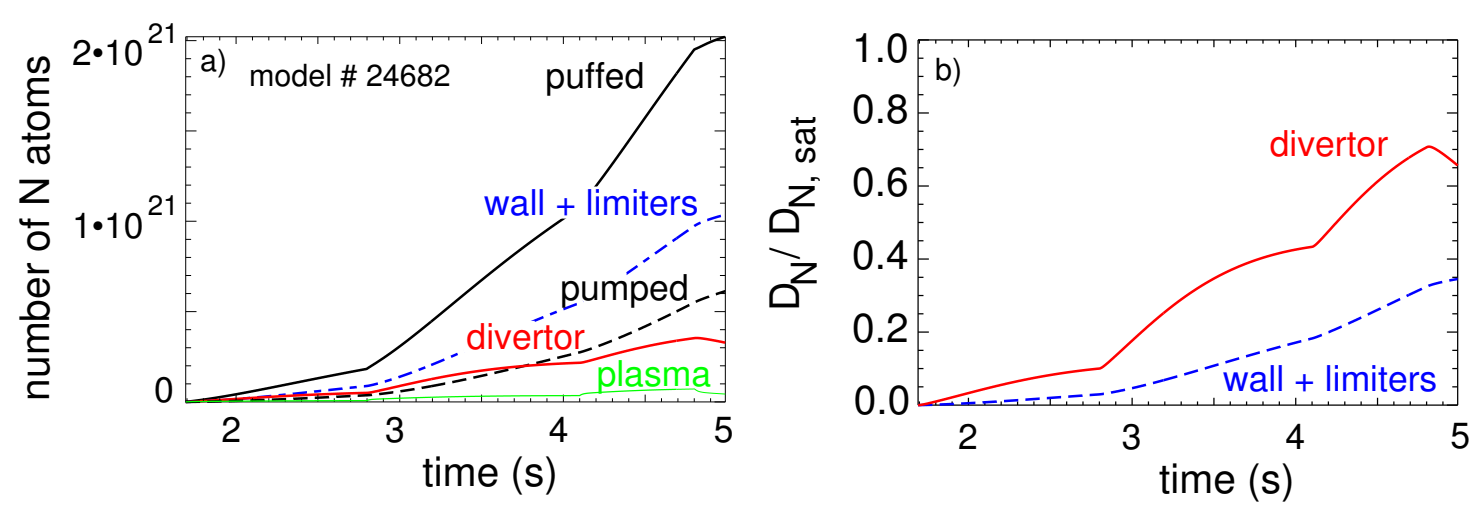

Figure 7. a) Nitrogen particle balance obtained from the response model for the seeded discharge 24682 from figure 6. b) Corresponding degree of wall saturation.

The result of the wall sticking model of the nitrogen particle balance of the seeded discharge from figure 6 is shown in figure 7 . At the end of the seeding phase, about $2 / 3$ of the injected nitrogen atoms stick to walls and divertor surfaces. The plasma content appears to play a negligible role in the particle balance. Even if an uncertainty of the nitrogen pumping rate of $\pm 50 \%$ caused by an uncertainty of the enrichment factor $\eta_{N}$ is taken into account, the nitrogen wall storage remains significant. According to the model, the nitrogen uptake by the divertor surface starts to saturate at the end of the pulse. The fact that the wall sticking saturates manifests in the observation that the nitrogen valve flux reduces after some time with constant heating power. If the saturation density is artificially increased in the model to $\mathrm{D}_{N, \text { sat }}=510^{21}$ atoms $/ \mathrm{m}^{2}$, a continuous rise of the nitrogen valve flux through all heating power levels is obtained, in contradiction to the experimental behaviour shown in figure 6 .

The deconvolution of bolometer measurements has shown that the additional radiation of nitrogen occurs predominantly in the divertor region. Although there is a significant rise of $\mathrm{Z}_{\text {eff }}$ in the core plasma, its main chamber radiation is offset by a reduced radiation caused by other low- $Z$ impurities. Figure 8 shows reduced main chamber radiation by carbon and oxygen with nitrogen seeding, which is predominantly due to the reduced contribution of ELMs to the radiative losses. The total VUV 

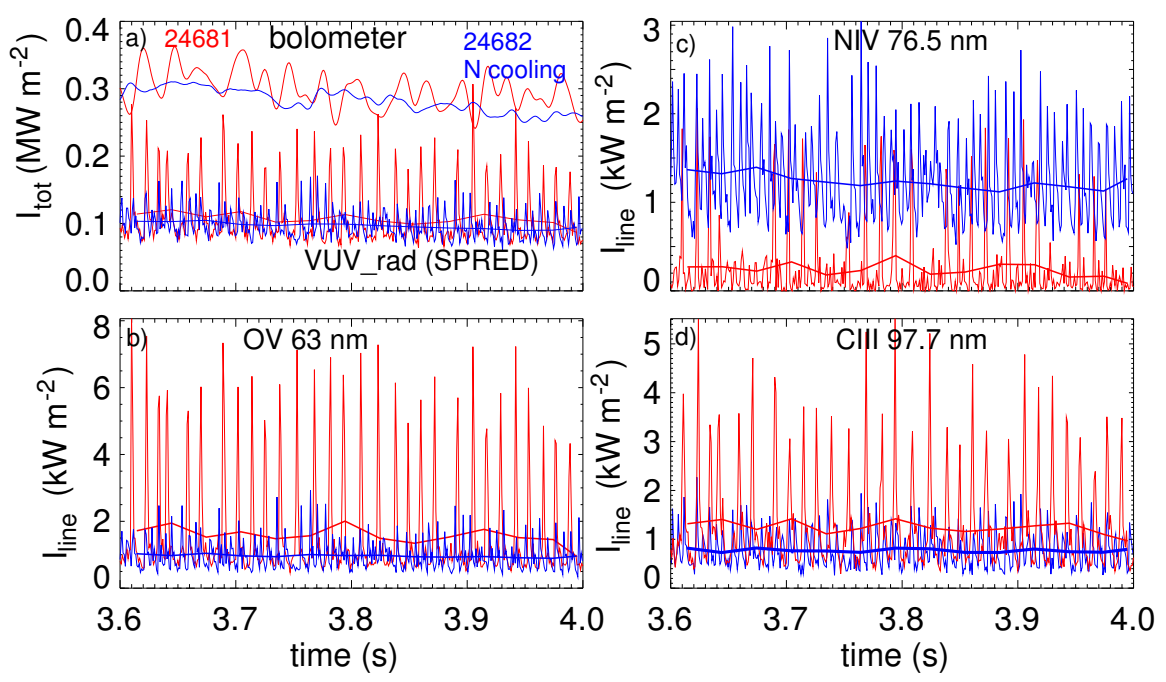

Figure 8. Comparison of the 2 discharges from figure 6 with (blue) and without (red) nitrogen seeding: a) Total line integrated radiation from bolometry and total VUV contribution measured by the SPRED spectrometer for radial viewing chords in the outer midplane. The VUV contribution corresponds to the spectral integral of the SPRED spectrometer from 12 to $118 \mathrm{~nm}$, with the $2^{\text {nd }}$ order contribution subtracted. The solid lines are temporally smoothed spectroscopic signals to show the ELM-averaged radiation level. b)-d) show the contribution for OV, NIV and CIII lines measured by the SPRED.

radiation as well as bolometry show about the same main chamber midplane radiation, the additional nitrogen radiation just offsets the reduced intrinsic ELM induced radiation losses.

The seeding of nitrogen has no significant effect on the erosion of tungsten and the plasma tungsten content. This is demonstrated in figure 9, where the tungsten concentration in the central plasma and the eroded neutral tungsten influxes are shown for the pair of discharges of figure 6 . Since the additional nitrogen is expected to contribute significantly to the eroded tungsten flux, this is offset by the reduced edge temperature caused by the nitrogen radiation. The only visible effect is a higher divertor tungsten erosion during ELMs for the nitrogen seeded case. Since the ELMs are shorter and have a reduced energy, the additional erosion by nitrogen ions must overcompensate for these effects. A reduction of the tungsten erosion yield caused by the high nitrogen concentration in the near surface layer as proposed in [34] is not visible in these data, but could be masked by a high rate of tungsten sputtering by nitrogen.

Figure 10 shows a discharge pair after boronisation with identical external parameters compared to the discharges in figure 6 . The feedback system is run with the optimized gains finally determined in the autumn 2009 campaign. Initialization at switch-on is done by setting the integral part to cancel the proportional part, resulting in zero gas flux at switch-on. The nitrogen consumption of the boronised discharge is about a factor of 2 larger than the unboronised reference. This value is larger than what 


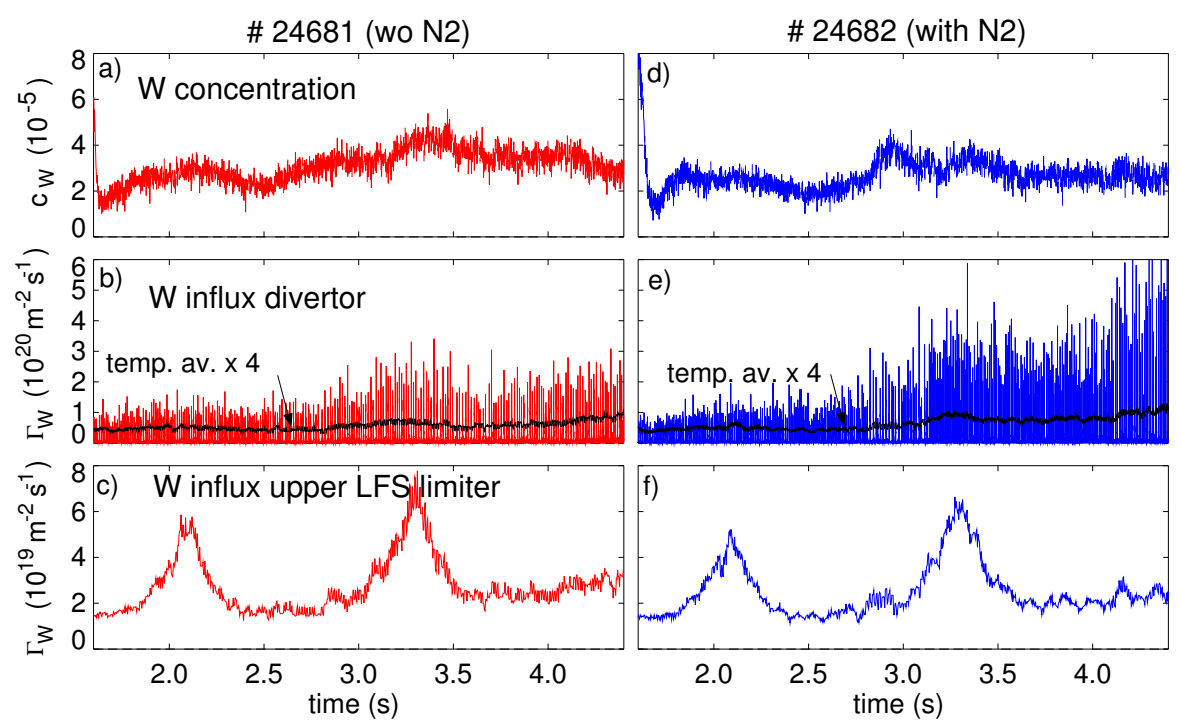

Figure 9. Comparison of the 2 discharges from figure 6 with and without nitrogen seeding. a), d) tungsten concentration in the core plasma from VUV spectroscopy and $W^{0}$ influx derived from the neutral WI line at $400.8 \mathrm{~nm}$. b), e) Fast, ELM resolving measurement ( $0.2 \mathrm{~ms}$ integration time) showing the outer divertor tungsten influx. c), f) Tungsten influx from the upper part of a low field side limiter. The two excursions in the limiter tungsten influx are caused by radial plasma sweeps towards the limiter, due to the low time resolution ELMS are not time resolved.

is expected from the slightly lower intrinsic radiation level. Therefore, a higher nitrogen wall storage level of the boron-coated tungsten is assumed. In the simulation model, the effect of the boronisation can be approximated by an increase of the saturation areal density $\mathrm{D}_{N, \text { sat }}$ by a factor of 3 . The nitrogen latency in subsequent discharges exhibits no significant difference in comparison to the unboronised discharges.

The nitrogen seeded discharge for the boronised pair of figure 10 shows a significantly higher stored energy compared to the weakly seeded discharge (see figure $10 \mathrm{~b})$. The enhancement of $\mathrm{H}_{98(y, 2)}$ goes up to a factor 1.3 , with stationary values of $\mathrm{H}_{98(y, 2)}=1.1$ with nitrogen and 0.9 without nitrogen around $\mathrm{t}=5 \mathrm{~s}$. The neutron rate is also enhanced, albeit roughly similar values are measured at the end of the seeded phase when the stored energy has somewhat relaxed. Nitrogen causes no change in the density profile shape, but a significant rise of core ion and electron temperatures is observed. Currently, this is thought to be due to a steepening of the critical gradients for turbulent transport due to the rise in $\mathrm{Z}_{\text {eff }}$ [37]. Dilution is not expected to be a key player here, since plasmas with high helium content usually show reduced confinement. The change of the ELM behaviour is thought not to be the origin of the improved confinement, since the smaller and shorter ELMs are already observed shortly after the nitrogen puff has started, and well before the improved confinement develops. The higher pedestal collisionality caused by the nitrogen induced $\mathrm{Z}_{\text {eff }}$ rise in the pedestal region is the primary suspect. 

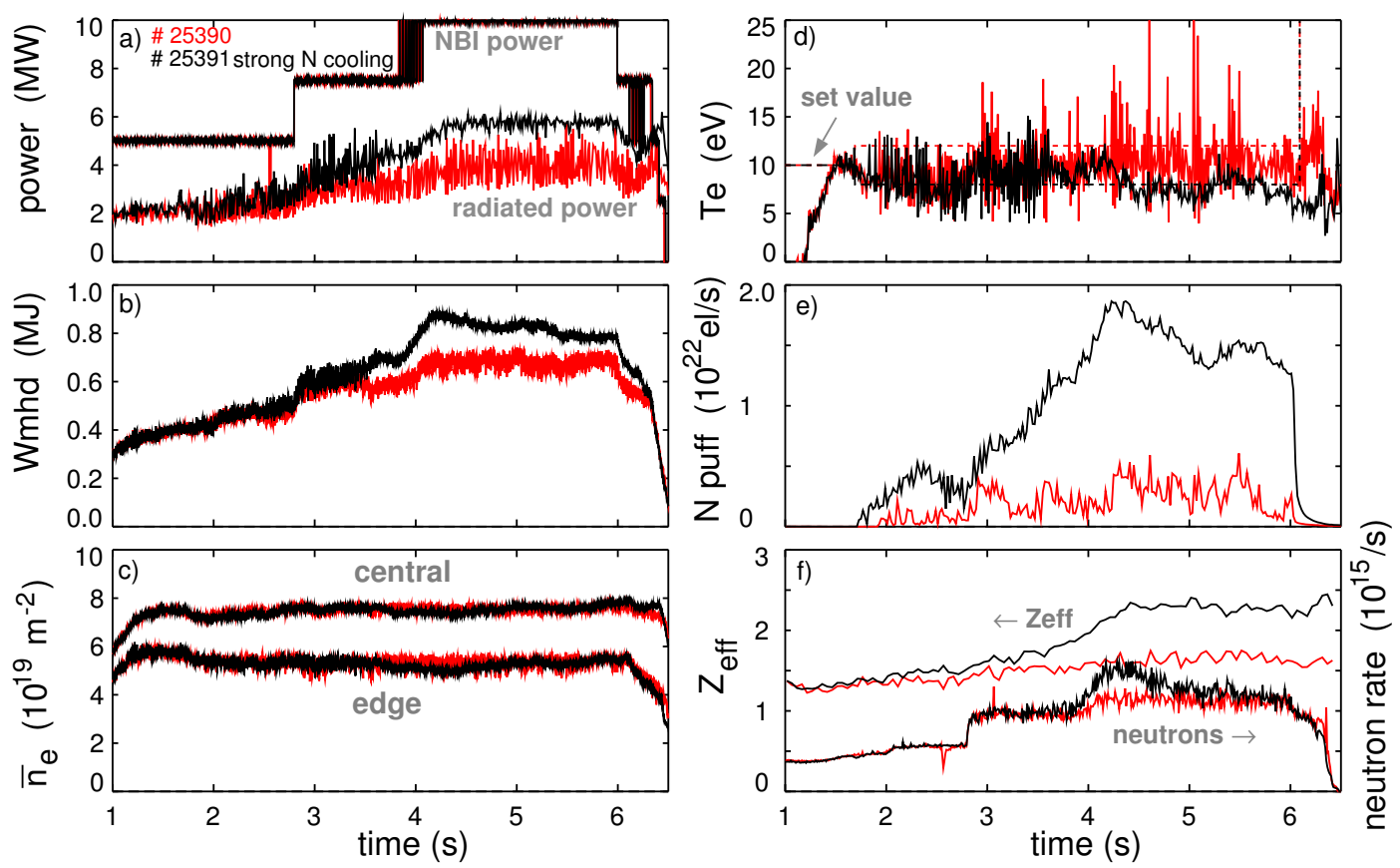

Figure 10. Comparison of 2 boronised discharges with weak (red) and strong (black) nitrogen seeding. Heating power and deuterium puff rate are identical to the unboronised discharge pair in figure 6. Feedback gains are set to the optimized values used since autumn 2009: $K_{p}=-810^{20} \mathrm{el} / \mathrm{s} / \mathrm{eV}, K_{i}=-810^{21} \mathrm{el} / \mathrm{s} /(\mathrm{s} \cdot \mathrm{eV})$.

\section{Optimisation of gain coefficients for routine operation}

While the model described above is not expected to allow precise predictions of impurity levels etc., it contains all relevant elements for a reasonably realistic simulation of the radiative feedback. This enables us to better understand and hence optimise the feedback system. E.g., inspection of figure 3 shows a fast reaction to the heating power step at $\mathrm{t}=2.8 \mathrm{~s}$, but a relatively slow approach of the set value after switching on the feedback.

Figure 11 shows the simulated behaviour of the feedback for the experimental conditions of figure 6 for different settings of the gain coefficients. In a), the standard values for the autumn 2009 experimental campaign are used, as applied to the discharge shown in figure 10. In b), the predicted behaviour for the assumption of zero nitrogen wall sticking is shown. The nitrogen valve flux required to obtain the same divertor heat flux is about a factor 3 lower compared to the case with wall sticking. Figures c)-e) exemplify the deficiencies of gain selections significantly different from the optimised choice, in line with the discussion in section 3. A reduced proportional gain as in c) results in a delayed reaction on fast transients. An increased integral gain in d) leads to increased valve fluxes and may lead to oscillatory behaviour and $T_{\text {div }}$ undershoot, which could cause an H-L transition. An increased proportional gain gives fast reaction to transients, but a relatively slow approach of the set value due to a long time constant 


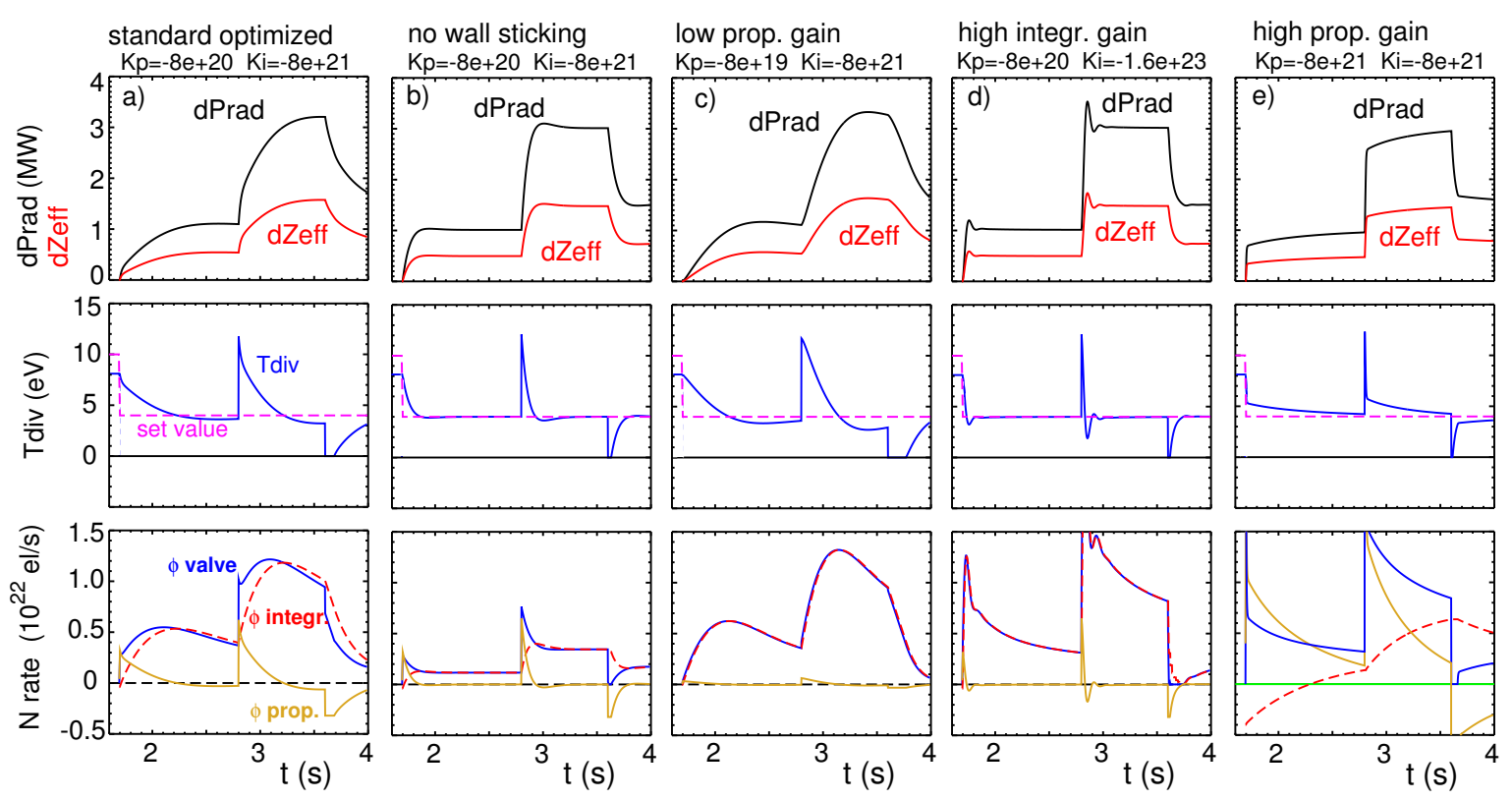

Figure 11. Modelled nitrogen feedback behaviour using the conditions similar to discharge \# 24682, figure 6. a) standard optimized gain set, b) same, but assuming zero wall nitrogen sticking, c) reduced proportional gain, d) increased integral gain, e) increased proportional gain.

$\mathrm{K}_{p} / \mathrm{K}_{i}$

\section{Conclusions}

The radiative target power load control in ASDEX Upgrade, which became necessary after complete tungsten coating of the plasma facing components, has beeen matured into a routine system. A simple 0-D plasma response model has been developed that allows us to better understand and optimise the system. The parameter variations applied in simulations using the simple plasma model corroborated the criteria for defining the gain coefficients described in section 3. The system uses a passive current measurement in divertor tiles as a feedback signal. Alternatively, a fast, online IR thermography system could be used to supply a direct measurement of the power load. The advantage of the shunt current measurement is its technical simplicity and robustness, which is an advantage for a system relevant for machine safety under high heating power conditions. A set of gains and initialization conditions has been found, that provides a fast and stable approach to the desired low target power load conditions for a wide range of heating powers.

Wall storage of nitrogen in tungsten was found to have a significant influence on the gas fluxes requested by the feedback system, as well as on the nitrogen content of subsequent discharges. The qualitative behaviour of nitrogen storage was found to be similar to that obtained in lab experiments under almost ideal conditions [34]. For the 
tokamak case, however, an order of magnitude larger saturation density of nitrogen in tungsten had to be assumed to explain the nitrogen particle balance. This is possibly explained by the much higher surface roughness in the tokamak, which leads to a higher effective surface area, or by other nitrogen retaining impurities (boron, carbon) situated in the micro-indentations associated with the surface roughness.

Future devices with power fluxes of several hundred megawatts will rely on strong radiative power removal. Feedback control schemes like that presented in this paper will be able to regulate the radiative power removal within the physically achievable boundaries. Since high impurity wall fluxes are expected in particular at the divertor targets, possible modifications of the near surface layer caused by implanted impurities need to be analysed for their effect on material properties and erosion rates.

\section{References}

[1] PACHER, G. W. et al., Nucl. Fusion 47 (2007) 469.

[2] TOBITA, K. et al., Nucl. Fusion 49 (2009) 075029.

[3] LAZARUS, E. A. et al., Nucl. Fusion 25 (1985) 135.

[4] MESSIAEN, A. M. et al., Nucl. Fusion 34 (1994) 825.

[5] GRUBER, O. et al., Phys. Rev. Lett. 74 (1995) 4217.

[6] JACKSON, G. L. et al., Nucl. Fusion 42 (2002) 28.

[7] HIGASHIJIMA, S. et al., J. Nucl. Mater. 313-316 (2003) 1123.

[8] TAKENAGA, H. et al., Nucl. Fusion 45 (2005) 1618.

[9] GOETZ, J. A. et al., Phys. Plasmas 6 (1999) 1899.

[10] PETRIE, T. W. et al., Nucl. Fusion 48 (2008) 045010.

[11] MATTHEWS, G. F. et al., Nucl. Fusion 39 (1999) 19.

[12] ONGENA, J. et al., Nucl. Fusion 44 (2004) 124.

[13] RAPP, J. et al., Nucl. Fusion 44 (2004) 312.

[14] MONIER-GARBET, P. et al., Nucl. Fusion 45 (2005) 1404.

[15] DUMORTIER, P. et al., Plasma Phys. Controlled Fusion 44 (2002) 1845.

[16] MADDISON, G. P. et al., Nucl. Fusion 43 (2003) 49.

[17] ASAKURA, N. et al., Nucl. Fusion 49 (2009) 115010.

[18] KALLENBACH, A. et al., Nucl. Fusion 35 (1995) 1231.

[19] NEU, R. et al., J. Nucl. Mater. 363-365 (2007) 52.

[20] KALLENBACH, A. et al., Nucl. Fusion 49 (2009) 045007.

[21] MADDISON, G. et al., Impurity-seeding experiments on jet in preparation of the ITER-like wall, in Europhysics Conference Abstracts (Proc. of the 36th EPS Conference on Controlled Fusion and Plasma Physics, Sofia, 2009), volume 33E, pages P-2.160, 2009.

[22] GRUBER, O. et al., Nucl. Fusion 49 (2009) 115014.

[23] KALLENBACH, A. et al., J. Nucl. Mater. 290-293 (2001) 639.

[24] STAEBLER, G. M. and HINTON, F. L., Nucl. Fusion 29 (1989) 1820.

[25] GIANNONE, L. et al., 'Data aquisition and real time signal processing of plasma diagnostics on ASDEX Upgrade using LabVIEW RT', submitted to Fusion Engineering and Design (2009).

[26] TREUTTERER, W. et al., Fusion Eng. Design 81 (2006) 1927.

[27] KAllenBACH, A. et al., Fusion Eng. Design 36 (1997) 101.

[28] KALLENBACH, A. et al., Nucl. Fusion 35 (1995) 1231.

[29] SCHNEIDER, R. et al., J. Nucl. Mater. 241-243 (1997) 701.

[30] DUX, R. et al., Plasma Phys. Controlled Fusion 38 (1996) 989, preprint in IPP 10/1, Febuary 1996.

[31] MATTHEWS, G. F. et al., J. Nucl. Mater. 241-243 (1997) 450. 
[32] DUX, R. and PEETERS, A. G., Nucl. Fusion 40 (2000) 1721.

[33] LACKNER, K., Comments Plasma Phys. Controlled Fusion 15 (1994) 359.

[34] SCHMID, K. et al., Nucl. Fusion 50 (2010) 025006.

[35] KALLENBACH, A. et al., Nucl. Fusion 43 (2003) 573.

[36] KALleNBACH, A. et al., Nucl. Fusion 42 (2002) 1184.

[37] TARDINI, G. et al., Confinement enhancement in ASDEX Upgrade improved H-mode discharges with nitrogen seeding, in Europhysics Conference Abstracts (Proc. of the 36th EPS Conference on Controlled Fusion and Plasma Physics, Sofia, 2009), volume 33E, pages O-2.004, 2009. 